\title{
A Case of Pyogenic Spondylitis after Surgery for Oral Floor Cancer
}

\author{
Takamitsu Mano*, Ryota Yamanouchi, Jutaro Miyamoto, Hirotsugu Umeda, \\ Yoshiya Ueyama \\ Department of Oral and Maxillofacial Surgery, Yamaguchi University Graduate School of Medicine, \\ Yamaguchi, Japan \\ Email: tmano@yamaguchi-u.ac.jp
}

Received 3 April 2014; revised 5 May 2014; accepted 12 May 2014

Copyright @ 2014 by authors and Scientific Research Publishing Inc.

This work is licensed under the Creative Commons Attribution International License (CC BY). http://creativecommons.org/licenses/by/4.0/

(c) (i) Open Access

\begin{abstract}
We report the case of a patient who developed pyogenic spondylitis after surgery for oral floor cancer. This 85-year-old man was evaluated at our hospital for a mass in the left floor of the mouth. Oral floor cancer (T2NOMO) was diagnosed. Surgical resection of the tumor was performed twice. On postoperative day 14, he developed sudden neck pain, followed by fever the next day and a gradual decrease in blood pressure. Septic shock was diagnosed, and echocardiography was performed, but no evidence of infective endocarditis was found. Findings on neck magnetic resonance imaging suggested pyogenic spondylitis, and ultimately pyogenic spondylitis causing septic shock was diagnosed. In patients with a compromised immune system after surgery who develop neck pain and fever, the possibility of pyogenic spondylitis as a complication should be kept in mind.
\end{abstract}

\section{Keywords}

Pyogenic Spondylitis, Oral Cancer, Sepsis

\section{Introduction}

Pyogenic spondylitis (PS) is a rapidly progressive inflammatory disease of the vertebrae with neurological symptoms such as quadriplegia. PS most commonly arises due to hematogenous spread of infection, but PS by direct spread of infection due to a retropharyngeal abscess, stellate ganglion block, or discography has also been reported [1]-[3]. The incidence of PS has recently increased due to the emergence of resistant bacteria and the increased number of immunocompromised hosts associated with the aging of society [4]. We now report the

\footnotetext{
*Corresponding author.
} 
case of a patient who developed PS, probably due to hematogenous infection, after undergoing surgery for oral floor cancer.

\section{Case Report}

This 85-year-old man noticed pain in the left floor of the mouth from October 2011. After no improvement was seen over time, the patient saw a local ear, nose and throat (ENT) specialist in December 2011. A mass was noted in the left floor of the mouth, and biopsy was performed. This revealed squamous cell carcinoma, so he was referred during that same month to our hospital.

Examination of the oral cavity revealed a $35 \times 18 \mathrm{~mm}$ indurated exophytic mass in the left oral floor (Figure 1). Contrast-enhanced CT showed no significant cervical lymphadenopathy. FDG-PET revealed uptake only in the left floor of the mouth, without any findings suggesting distant metastases. Left oral floor cancer (T2NOM0) was diagnosed, and tumor resection was performed in January 2012. Anteriorly and posteriorly, the wound was closed with several absorbable sutures and covered with a polyglycolic acid (PGA) sheet attached with fibrin glue.

In March, tumor recurrence was noted in the left retromolar triangle, so resection was again performed. As during the first surgery, the wound was covered with a PGA sheet attached using fibrin glue. On postoperative day 10, although the wound was not completely epithelialized, healing was good (Figure 2). However, on post

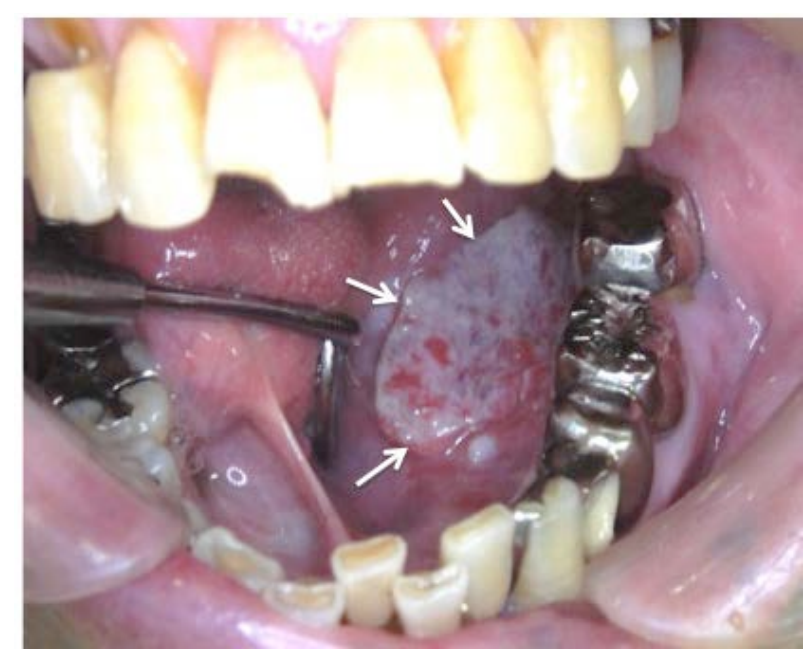

Figure 1. The oral cavity preoperatively. A $35 \times 18 \mathrm{~mm}$ indurated exophytic mass is seen in the left oral floor (arrows).

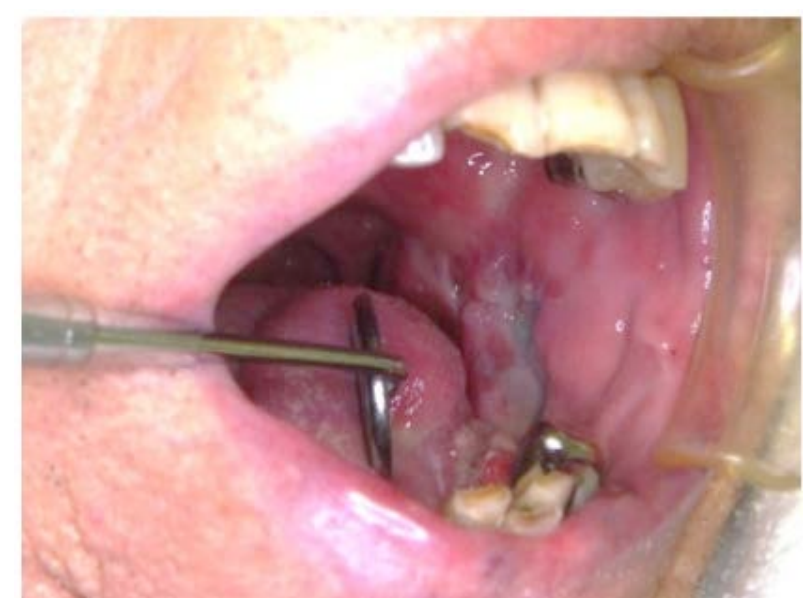

Figure 2. The oral cavity after repeat surgery. The wound is not yet completely healed, but no signs of infection are present. 
operative day 14, the patient experienced sudden right posterior neck pain. An orthopedic consultant recommended analgesics, topical agents, and follow-up observation.

The next day, serum C-reactive protein (CRP) level rose to $7.61 \mathrm{mg} / \mathrm{dl}$, and the patient had a fever exceeding $40^{\circ} \mathrm{C}$, so blood cultures were drawn. No indwelling urinary catheter or intravenous line was in use at this time. Chest radiography showed no inflammation, but the possibility of aspiration pneumonia was considered, and an antibiotic (ciprofloxacin; CPFX) was started. A rapid influenza test yielded negative results. The following day, blood pressure dropped to 70/36 mmHg, and blood pressure did not rise despite fluid replacement. Septic shock was therefore suspected, and the patient was transferred to the intensive care unit (ICU).

As blood cultures were positive for Streptococcus spp., infective endocarditis was suspected, and a cardiologist recommended transesophageal echocardiography. However, this showed no abnormalities, ruling out infective endocarditis. Antibiotic treatment was switched to sulbactam sodium/ampicillin sodium (ABPC/SBT) and cefotaxime sodium (CTX). Three days after symptom onset, CRP increased to $10.1 \mathrm{mg} / \mathrm{dl}$. Although no neurological symptoms were identified, PS was suspected, and neck magnetic resonance imaging (MRI) was performed. T2-weighted imaging showed fluid accumulation anterior to the vertebral bodies at the C2 - C6 level and intramedullary signal hyperintensity at C4 and C5. PS was therefore diagnosed (Figure 3).

The orthopedic consultant recommended a neck brace. MRI and CT also showed inflammation of the paranasal sinuses, so the possibility of PS due to spread of a retropharyngeal abscess was also considered, and an ENT specialist at our hospital examined the patient. Endoscopy was performed, but showed no retropharyngeal abscess or other abnormality.

On day 4 after symptom onset, blood pressure was stable at 104/62 mmHg, CRP decreased to $8.25 \mathrm{mg} / \mathrm{dl}$, and the patient was transferred back from the ICU to the general ward. Ultimately, blood cultures were positive for Streptococcus agalactiae and treatment was switched to ampicillin sodium (ABPC) based on antibiotic sensitivity. Cultures of the oral cavity yielded positive results only for Candida albicans and alpha-hemolytic Streptococcus, but Streptococcus agalactiae was not detected. Before and after the onset of neck pain, no abdominal symptoms such as abdominal pain or diarrhea were seen.

On day 13 after symptom onset, CRP decreased to $1.23 \mathrm{mg} / \mathrm{dl}$, and the patient was transferred to the ortho-

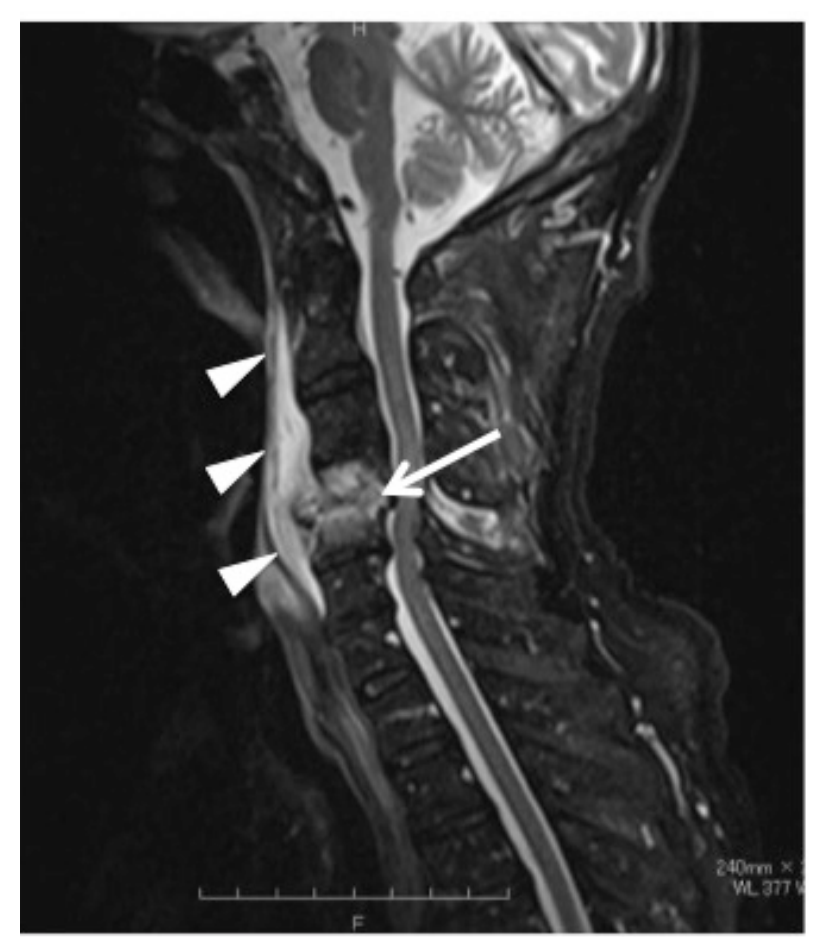

Figure 3. Neck MRI (T2-weighted imaging). Fluid accumulation is seen anterior to the vertebral bodies at the C2 - C6 level (arrowheads) and intramedullary signal hyperintensity at C4 and C5 (arrow). 
pedic surgery ward for further treatment of PS. Antibiotic treatment was switched to cefazolin sodium (CEZ). Repeat MRI showed enlargement of the neck abscess, so long-term treatment with meropenem (MEPM) was started (Figure 4). Subsequently, the patient had a fall in the hospital ward and became quadriplegic. Despite long-term antibiotic therapy, CRP levels did not normalize. The patient was transferred in June to another facility for rehabilitation. As of the time of writing, no recurrence of the oral cancer or any metastases has been seen. However, the patient remains quadriplegic and requires use of a wheelchair.

\section{Discussion}

PS is an infection directly under the vertebral body endplate adjacent to the intervertebral disk, and can spread to the center of the vertebral body and the intervertebral disk. PS most often develops in the lumbar spine, followed by the thoracic spine, but PS of the cervical spine is relatively uncommon, accounting for $5 \%-15 \%$ of all cases [3] [5] [6].

PS was previously considered a rare disease, but with the emergence of resistant bacteria and increasing numbers of compromised hosts associated with the aging of society, the incidence of PS is increasing. PS occurs more often in patients with diabetes, rheumatoid arthritis, long-term steroid use, malignant tumors, and chronic renal or liver diseases [4]. Males appear to be affected twice as often as females though the reason for this is unknown [7]. PS most commonly spreads by a hematogenous route of infection, and hematogenous infection from pneumonia, as well as hematogenous infection in cases where the primary focus of infection is unknown, has been reported [4] [8] [9]. The arterial route of spread is more common than the venous route, usually from skin, respiratory tract, genitourinary tract, gastrointestinal tract or the oral cavity, giving rise to bacteraemia [8]. Direct spread of infection may also occur, for example, from a retropharyngeal abscess, or after stellate ganglion block or discography [1]-[3]. However, to the best of our knowledge, PS after oral surgery has not previously been reported. The patient in this case had no history of diabetes or retropharyngeal abscess. We speculate that our patient was an advanced age and had decreased immunocompetence after oral cancer surgery, and that PS occurred by hematogenous infection.

The causative organisms in PS include the normal oral and intestinal bacterial flora such as Staphylococcus aureus, Streptococcus agalactiae, Eschrichia coli, Streptococcus viridians, and Streptococcus oralis [8] [10]. It was reported that most PS patients due to Streptococcus agalactiae were male, between 50 and 70 years of age, with predisposing conditions such as diabetes mellitus or neoplasia [10]. Blood cultures in our patient were positive for Streptococcus agalactiae. This is also known as Group B Streptococcus, is found in the intestinal and vaginal normal flora, and is a clinically important cause of neonatal septicemia and meningitis. Bacterial-cultures in our patient did not detect Streptococcus agalactiae from the oral cavity, so PS was probably caused by hematogenous infection from intestinal bacteria.

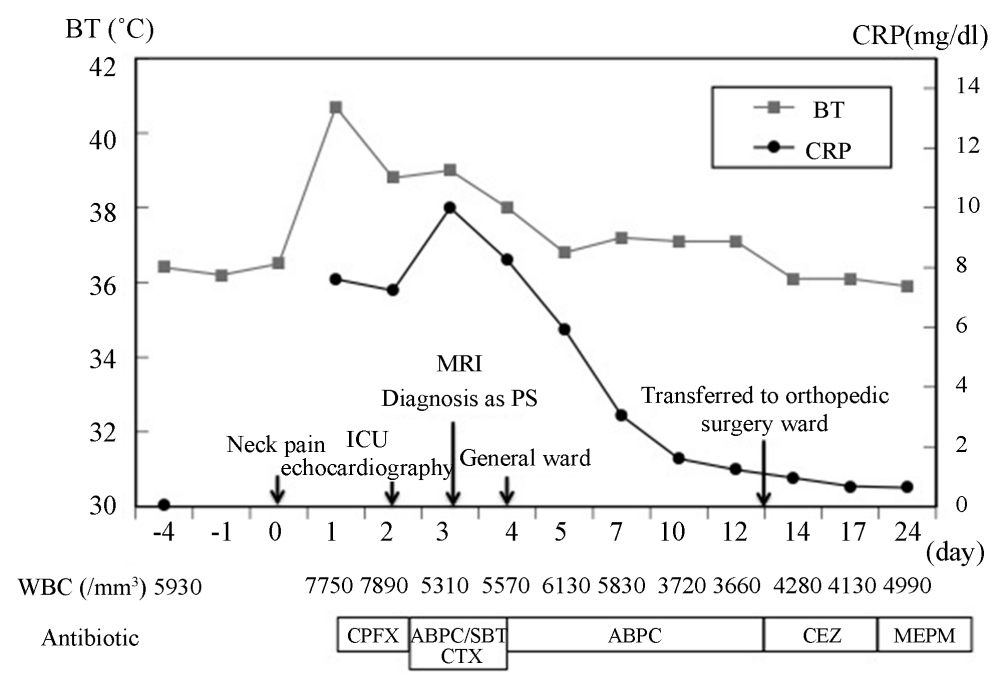

Figure 4. Treatment and clinical course. Changes in treatment, maximum temperature, CRP, and WBC count (onset of neck pain defined as day 0 ). 
Early PS is associated with few specific findings on plain cervical spine radiography, but as PS progresses, vertebral body atrophy, destruction of the vertebral body margin, narrowing of the intervertebral disk, prevertebral soft tissue swelling, and gradual destruction of the vertebral body may be observed. MRI is useful for the diagnosis of PS, provides soft tissue information, and can clearly depict the site and extent of any spinal cord compression [10].

PS is usually treated conservatively with neck stabilization and antibiotics, but surgery is indicated for extensive bony destruction or severe neurological symptoms [3]. Our patient was treated conservatively with local stabilization using a neck brace and antibiotic therapy. However, with the addition of the falling incident, he is now quadriplegic and has residual disability.

\section{Conclusion}

PS is a disease that is almost unknown to occur in association with oral surgery, but as in our patient, may occur postoperatively due to decreased immune competence. Therefore, if septicemia occurs, PS should be considered alongside catheter infections and infective endocarditis during further evaluation. Moreover, the possibility of PS should be kept in mind in patients with neck pain or quadriplegia symptoms associated with fever, and further evaluation is essential.

\section{References}

[1] Faidas, A., Ferguson Jr., J.V., Nelson, J.E. and Baddour, L.M. (1994) Cervical Vertebral Osteomyelitis Presenting as a Retropharyngeal Abscess. Clinical Infectious Diseases, 18, 992-994. http://dx.doi.org/10.1093/clinids/18.6.992

[2] Tasdemiroglu, E., Sengöz, A. and Bagatur, E. (2004) Iatrogenic Spondylodiscitis. Case Report and Review of Literature. Neurosurgical Focus, 16, 1-5.

[3] Korovessis, P., Repantis, T. and Hadjipavlou, A.G. (2012) Hematogenous Pyogenic Spinal Infection: Current Perceptions. Orthopedics, 35, 885-892. http://dx.doi.org/10.3928/01477447-20120919-11

[4] Fantoni, M., Trecarichi, E.M., Rossi, B., Mazzotta, V., Di Giacomo, G., Nasto, L.A., Di Meco, E. and Pola, E. (2012) Epidemiological and Clinical Features of Pyogenic Spondylodiscitis. European Review for Medical and Pharmacological Sciences, 16, 2-7.

[5] Messer, H.D. and Litvinoff, J. (1976) Pyogenic Cervical Osteomyelitis. Archives of Neurology, 33, 571-576. http://dx.doi.org/10.1001/archneur.1976.00500080049007

[6] Eismont, F.J., Bohlman, H.H., Soni, P.L., Goldberg, V.M. and Freehafer, A.A. (1983) Pyogenic and Fungal Vertebral Osteomyelitis with Paralysis. Journal of Bone and Joint Surgery, 65A, 19-29.

[7] Tali, E.T. (2004) Spinal Infection. European Journal of Radiology, 50, 120-133. http://dx.doi.org/10.1016/j.ejrad.2003.10.022

[8] Cheung, W.Y. and Luk, K.D.K. (2012) Pyogenic Spondylitis. International Orthopaedics, 36, 397-404. http://dx.doi.org/10.1007/s00264-011-1384-6

[9] Morishita, S., Fujiwara, H., Murota, H., Maeda, Y., Hara, A. and Horii T. (2013) Bloodstream Infection Caused by Campylobacter Lari. Journal of Infection and Chemotherapy, 19, 333-337. http://dx.doi.org/10.1007/s10156-012-0471-y

[10] Solís-Garcia del Pozo, J., Martinez-Alfaro, E., Abad, L. and Solera, J. (2000) Vertebral Osteomyelitis Caused by Streptococcus agalactiae. Journal of Infection, 41, 84-90. http://dx.doi.org/10.1053/jinf.2000.0694 